Article

\title{
Low Body Weight Increases the Risk of Ischemic Stroke and Major Bleeding in Atrial Fibrillation: The COOL-AF Registry
}

\author{
Rungroj Krittayaphong ${ }^{1, *(1)}$, Ply Chichareon ${ }^{2}$, Chulalak Komoltri ${ }^{1}$, Sakaorat Kornbongkotmas ${ }^{3}$, \\ Ahthit Yindeengam ${ }^{1}$ and Gregory Y. H. Lip ${ }^{4,5,+}+$ on behalf of the COOL-AF Investigators \\ 1 Faculty of Medicine Siriraj Hospital, Mahidol University, Bangkok 10700, Thailand; \\ chulaluk.kom@mahidol.ac.th (C.K.); porpor_2@hotmail.com (A.Y.) \\ 2 Cardiology unit, Department of Internal Medicine, Faculty of Medicine, Prince of Songkla University, \\ Songkla 90110, Thailand; plyc83@yahoo.com \\ 3 Queen Savang Vadhana Memorial Hospital, Chonburi 20110, Thailand; hembae174@gmail.com \\ 4 Liverpool Centre for Cardiovascular Science, University of Liverpool and Liverpool Heart \& Chest Hospital, \\ Liverpool L14 3PE, UK; Gregory.Lip@liverpool.ac.uk \\ 5 Aalborg Thrombosis Research Unit, Department of Clinical Medicine, Aalborg University, \\ DK-9100 Aalborg, Denmark \\ * Correspondence: rungroj.kri@mahidol.ac.th; Tel.: +66-2-419-6104; Fax: +66-2-412-7412 \\ + Membership of the COOL-AF Investigators is provided in the Acknowledgments.
}

Received: 11 June 2020; Accepted: 20 August 2020; Published: 22 August 2020

check for updates

\begin{abstract}
We aimed to determine if low body weight (LBW) status $(<50 \mathrm{~kg})$ is independently associated with increased risk of ischemic stroke and bleeding in Thai patients with non-valvular atrial fibrillation (NVAF). (1) Background: It has been unclear whether LBW influence clinical outcome of patients with NVAF. (2) Methods: This prospective multicenter cohort study included patients enrolled in the COOL-AF Registry. The following data were collected: demographic data, medical history, risk factors and comorbid conditions, laboratory and investigation data, and medications. Follow-up data were collected every 6 months. Clinical events during follow-up were confirmed by the adjudication committee. (3) Results: A total of 3367 patients were enrolled. The mean age was $67.2 \pm 11.2$ years. LBW was present in 338 patients (11.3\%). Anticoagulant and antiplatelet was prescribed in $75.3 \%$ and $26.2 \%$ of patients, respectively. Ischemic stroke, major bleeding, intracerebral hemorrhage (ICH), and death occurred during follow-up in $2.9 \%, 4.4 \%, 1.4 \%$, and $7.7 \%$ of patients, respectively, during 25.7 months follow-up. LBW was an independent predictor of ischemic stroke, major bleeding, $\mathrm{ICH}$, and death, with a hazard ratio of 2.40, 1.79, 2.37, and 2.65, respectively. (4) Conclusions: LBW was independently associated with increased risk of adverse outcomes in Thai patients with NVAF. This should be carefully considered when balancing the risks and benefits of stroke prevention among patients with different body weights.
\end{abstract}

Keywords: low body weight; ischemic stroke; major bleeding; Thai patients; non-valvular atrial fibrillation; COOL-AF Registry

\section{Introduction}

Non-valvular atrial fibrillation (NVAF) can lead to the development of thromboembolism and subsequent ischemic stroke [1]. Practice guidelines for oral anticoagulation (OAC) management in patients with NVAF suggest that patients with a CHA2DS2-VASc score of 1 should be considered for OAC treatment [2-4]. 
The Asian population is at increased risk for developing ischemic stroke [5] and intracerebral hemorrhage [6,7]. Even patients with a CHA2DS2-VASc score of 0 may have an ischaemic stroke risk of $1.15 \%$ per year [8], leading to a modified CHA2DS2-VASc score being proposed for Asian patients [9]. In Asian patients, body weight may influence the risk of stroke [10], which is particularly relevant since Asian peoples are often of smaller stature and lower weight compared to white Europeans. Of note, being of low body weight (LBW) might increase stroke risk in both white European [11] and Asian populations [12]. LBW also increases the risk of bleeding, especially among those who are on OAC [12]. In those taking vitamin $\mathrm{K}$ antagonist (e.g., warfarin), the dose of warfarin to achieve the target international normalized ratio (INR) may be lower in LBW patients due to a smaller volume of drug distribution; however, the likelihood of the development of complications after drug dose adjustment was reported to be higher in LBW patients [13]. Dose adjustment of certain non-vitamin K antagonist oral anticoagulants (NOACs) is recommended in patients with a lower body weight [14].

The COOL-AF registry is a prospective nationwide registry of newly diagnosed patients with NVAF, conducted in multiple centers in Thailand, as previously described [15]. Given that many Thai patients are of LBW (typical of many Asian peoples), the aim of this study was to determine if LBW status $(<50 \mathrm{~kg})$ was independently associated with increased risk of ischemic stroke and bleeding in Thai patients with NVAF.

\section{Methods}

\subsection{Study Population}

In COOL-AF, we prospectively and consecutively enrolled patients aged 18 years or older with definitively diagnosed NVAF by 24-lead electrocardiograph (ECG) or Holter monitoring at one of 27 large hospitals located throughout Thailand. A list of investigators is shown in Supplemental materials. Patients with any of the following were excluded: mechanical heart valves; rheumatic valve disease; hematologic conditions that increase the risk of bleeding; NVAF from transient reversible causes; life expectancy less than 3 years; unable to attend follow-up visits; refusal to participate; ischemic stroke within the preceding 3 months; pregnant at the time of the eligibility assessment; and participation in a clinical trial at the time of the eligibility assessment. The Institutional Review Board (IRB) of each participating hospital approved the study protocol and all enrolled patients approved their participation by providing written informed consent.

\subsection{Study Protocol and Data Collection}

Each patient was fully informed about the purpose of and the details relating to the study. Patient data were collected from a review of patient medical records and from the patient interview. Required data were written in the case record form (CRF). Data from the CRF were then transferred into the web-based data collection and management system and the hard copy of the CRF was sent to the central data management unit. Upon receiving the hard copy of the CRF, the study team at the data management unit cross-checked the data on the form with the data entered into the web-based system. Discrepancies of any kind were addressed to the study team at that study site. When the patient data for each patient was determined to be correct and complete, the data were locked and could not be changed. Follow-up data were collected and reviewed in a similar manner. Data were collected every 6 months until the last visit at 3 years after enrollment.

The following data were collected at the baseline visit: demographic data; weight and height; vital signs; type, duration and symptoms of NVAF; risk factors, such as diabetes and hypertension; comorbidities, such as coronary artery disease (CAD), heart failure (HF), or stroke; and all current medications for medical illnesses and prescribed antithrombotic agents, including $\mathrm{OAC}$ and antiplatelet drugs. Each component of CHA2DS2-VASc and HAS-BLED score was also collected. Clinical events and changes in risk factors and/or medications were collected at each follow-up visit. 


\subsection{Outcome Measurement}

The evaluated clinical outcomes during follow-up were ischemic stroke or transient ischemic attack (TIA), major bleeding, intracerebral hemorrhage, and death. The research team at each study site was instructed to upload the source document into the web-based system to confirm the evidence of each event. All events were reviewed and validated by the study team at the data management unit. After validation, all data were sent to the adjudication committee for final evaluation and confirmation. In cases with inconclusive evidence or further questions, additional data or an explanation was requested from the study site. Ischemic stroke was defined as a sudden-onset neurologic deficit that persisted for longer than $24 \mathrm{~h}$. TIA was defined as a sudden-onset neurologic deficit that lasted not longer than $24 \mathrm{~h}$. International Society of Thrombosis and Haemostasis (ISTH) criteria [16] was used to define major bleeding, including fatal bleeding, bleeding in critical organs, bleeding that results in a decrease in hemoglobin level of $20 \mathrm{~g} / \mathrm{L}$ or more, or bleeding that requires a transfusion of 2 units of red cells or more.

\subsection{Statistical Analysis}

Descriptive statistics were used to summarize patient demographic and clinical characteristics. LBW was defined as a body weight less than $50 \mathrm{~kg}$. Mean \pm standard deviation was used to describe continuous data and number and percentage were used to present categorical data. Comparisons between patients with body weight $<50$ and $\geq 50 \mathrm{~kg}$ were performed using Student's $t$-test for unpaired continuous data and chi-square test for categorical data. Comparisons of clinical outcomes between and among groups were made using the chi-square test. Associations between LBW and clinical outcomes over time were tested by the Cox proportional hazards model. Kaplan-Meier estimate with log-rank test was performed to determine significant differences among groups. The results of that analysis are reported as hazard ratio (HR) and 95\% confidence interval (CI) and are graphically displayed as the cumulative event rate compared between groups. Adjusted HR and 95\% CI was also reported as Forest plot after adjustment for age, sex, comorbid conditions (CAD, HF, smoking status, diabetes, hypercholesterolemia, hypertension, history of ischemic stroke, history of major bleeding, renal replacement therapy, cardiac implantable electronic devices) and antithrombotic medications (OAC and antiplatelet). Generalized Estimating Equation (GEE) with exchangeable correlation structure was used to analyse the effect of LBW on clinical outcomes with the adjustment of time varying covariates. Cox regression models with restricted cubic splines were used to determine the association between body weight as a continuous variable and the rates of clinical outcomes adjusted for age, sex, and comorbid conditions. Net clinical benefit (NCB) of OAC versus no OAC was calculated using the formula: (ischemic stroke/TIA rate off OAC-ischemic stroke/TIA rate on OAC)-1.5 (ICH rate on OAC-ICH rate off OAC) [17]. NCB of NOAC versus warfarin was calculated in a similar manner. A $p$-value of $<0.05$ was considered statistically significant. All statistical analyses were performed using SPSS Statistics version 23 (SPSS, Inc., Chicago, IL, USA, www.ibm.com/analytics/spss-statistics-software), R version 3.6.3 (www.r-project.org), and SAS studio (SAS Institute Inc., Cary, NC, USA, www.sas.com/en_th/contact.html).

\section{Results}

We enrolled a total of 3367 patients (mean age $67.2 \pm 11.2$ years; $58.5 \%$ male) in this study. The average CHA2DS2-VASc and HAS-BLED scores were $3.05 \pm 1.68$ and $1.53 \pm 1.01$, respectively. The distributions of body weight among male patients, female patients, and those aged $\geq 65$ years and age $<65$ years are shown in Figure S1 in Supplemental Material. The average body weight was $66.1 \pm 14.5 \mathrm{~kg}$; of these, $381(11.3 \%)$ were classified as having LBW status. Table 1 shows baseline demographic and clinical characteristics compared between patients with body weight $<50$ and $\geq 50 \mathrm{~kg}$. Patients with LBW were significantly older; more likely to be female; less likely to have hypertension, 
diabetes, dyslipidemia, and be a current smoker; had higher CHA2DS2-VASc and HAS-BLED scores; and were less likely to use antiplatelet drugs.

Table 1. Baseline characteristics compared between patients with body weight $<50$ and $\geq 50 \mathrm{~kg}$.

\begin{tabular}{|c|c|c|c|c|}
\hline Characteristics & All $(n=3367)$ & BW $<50(n=380)$ & $\mathrm{BW} \geq 50(n=2987)$ & $p$ \\
\hline Age (years) & $67.2 \pm 11.2$ & $74.1 \pm 10.2$ & $66.4 \pm 11.1$ & $<0.001$ \\
\hline Female & $1397(41.5 \%)$ & $284(74.7 \%)$ & $1113(37.3 \%)$ & $<0.001$ \\
\hline Time after NVAF diagnosis (years) & $3.4 \pm 4.3$ & $3.2 \pm 3.9$ & $3.4 \pm 4.4$ & 0.362 \\
\hline Type of NVAF & & & & 0.070 \\
\hline - Paroxysmal & $1131(33.6 \%)$ & $115(30.3 \%)$ & $1016(34.0 \%)$ & \\
\hline - Persistent & $642(19.1 \%)$ & $64(16.8 \%)$ & $578(19.4 \%)$ & \\
\hline - Permanent & $1594(47.3 \%)$ & $201(52.9 \%)$ & $1393(46.6 \%)$ & \\
\hline Symptomatic NVAF & $2600(77.2 \%)$ & $292(76.8 \%)$ & $2308(77.3 \%)$ & 0.852 \\
\hline History of heart failure & $897(26.6 \%)$ & $93(24.5 \%)$ & $804(26.9 \%)$ & 0.310 \\
\hline History of coronary artery disease & $543(16.1 \%)$ & $44(11.6 \%)$ & $499(16.7 \%)$ & 0.010 \\
\hline CIED & $329(9.8 \%)$ & $45(11.8 \%)$ & $284(9.5 \%)$ & 0.149 \\
\hline History of ischemic stroke/TIA & $573(17.0 \%)$ & $72(18.9 \%)$ & $501(16.8 \%)$ & $<0.001$ \\
\hline Hypertension & $2299(68.3 \%)$ & $229(60.3 \%)$ & $2070(69.3 \%)$ & $<0.001$ \\
\hline Diabetes mellitus & $834(24.8 \%)$ & $44(11.6 \%)$ & $790(26.4 \%)$ & $<0.001$ \\
\hline Current smoker & $676(20.1 \%)$ & $34(8.9 \%)$ & $642(21.5 \%)$ & $<0.001$ \\
\hline Dyslipidemia & $1901(56.5 \%)$ & $163(42.9 \%)$ & $1738(58.2 \%)$ & $<0.001$ \\
\hline Renal replacement therapy & $38(1.1 \%)$ & $3(0.8 \%)$ & $35(1.2 \%)$ & 0.795 \\
\hline Dementia & $28(0.8 \%)$ & $4(1.1 \%)$ & $24(0.8 \%)$ & 0.549 \\
\hline History of bleeding & $322(9.6 \%)$ & $45(11.8 \%)$ & $277(9.3 \%)$ & 0.109 \\
\hline CHA2DS2-VASc score & & & & $<0.001$ \\
\hline-0 & $198(5.9 \%)$ & $4(1.1 \%)$ & $194(6.5 \%)$ & \\
\hline-1 & $422(12.5 \%)$ & $21(5.5 \%)$ & $401(13.4 \%)$ & \\
\hline$-\geq 2$ & $2747(81.6 \%)$ & $355(93.4 \%)$ & $2392(80.1 \%)$ & \\
\hline HAS-BLED score & & & & $<0.001$ \\
\hline-0 & $489(14.5 \%)$ & $25(6.6 \%)$ & $464(15.5 \%)$ & \\
\hline$-1-2$ & $2354(69.9 \%)$ & $281(73.9 \%)$ & $2073(69.4 \%)$ & \\
\hline$-\geq 3$ & $524(15.6 \%)$ & $74(19.5 \%)$ & $450(15.1 \%)$ & \\
\hline Antiplatelet & $882(26.2 \%)$ & $81(21.3 \%)$ & $801(26.8 \%)$ & 0.022 \\
\hline Anticoagulant & $2535(75.3 \%)$ & $293(77.1 \%)$ & $2242(75.1 \%)$ & 0.384 \\
\hline - Warfarin & $2310(68.6 \%)$ & $276(72.6 \%)$ & $2034(68.1 \%)$ & 0.073 \\
\hline - NOACs & $225(6.7 \%)$ & $17(4.5 \%)$ & $208(7.0 \%)$ & 0.067 \\
\hline \multicolumn{5}{|l|}{ For warfarin group } \\
\hline - Time in therapeutic range (\%) & $53.5 \pm 26.4$ & $53.0 \pm 26.2$ & $53.6 \pm 26.4$ & 0.722 \\
\hline - Time under therapeutic range (\%) & $32.1 \pm 27.6$ & $29.9 \pm 26.2$ & $32.4 \pm 27.8$ & 0.158 \\
\hline - Time above therapeutic range (\%) & $14.1 \pm 17.5$ & $17.0 \pm 19.9$ & $13.7 \pm 17.1$ & 0.010 \\
\hline - Baseline INR & $2.2 \pm 0.8$ & $2.4 \pm 0.9$ & $2.2 \pm 0.8$ & $<0.001$ \\
\hline
\end{tabular}

Data presented as mean \pm standard deviation or number and percentage; A $p$-value $<0.05$ indicates statistical significance. Abbreviations: BW, body weight; NVAF, non-valvular atrial fibrillation; CIED, cardiac implantable electronic device; TIA, transient ischemic attack; NOACs, non-vitamin $\mathrm{K}$ antagonist oral anticoagulants; INR, international normalized ratio

\subsection{LBW and Outcomes}

The average follow-up duration was $25.7 \pm 10.6$ months. Among the main clinical outcomes, ischemic stroke/TIA, major bleeding, ICH, and death occurred in $99(2.9 \%), 148(4.4 \%), 48(1.4 \%)$, and $260(7.7 \%)$ patients, respectively.

LBW increased the risk of ischemic stroke, major bleeding, $\mathrm{ICH}$, and death with a hazard ratio (HR; 95\% confidence interval (CI)) of 2.40 (1.51-3.83), 1.79 (1.18-2.72), 2.37 (1.21-4.65), and 2.65 (1.99-3.53), respectively. Rate of ischemic stroke/TIA, major bleeding, ICH, and death were 3.20, 3.78, 1.53, and 8.76 per 100 persons-year for low BW and 1.19, 1.89, 0.58, and 3.08 per 100 persons-year for non-LBW, respectively. The cumulative event rate is graphically shown for all four clinical outcomes compared between patients with body weight $<50$ and $\geq 50 \mathrm{~kg}$ in Figure 1. These graphs show a statistically significant difference between body weight groups for all four clinical outcomes and the difference in the number of events continued to widen over the follow-up duration for all 4 clinical event subgroups. 
A

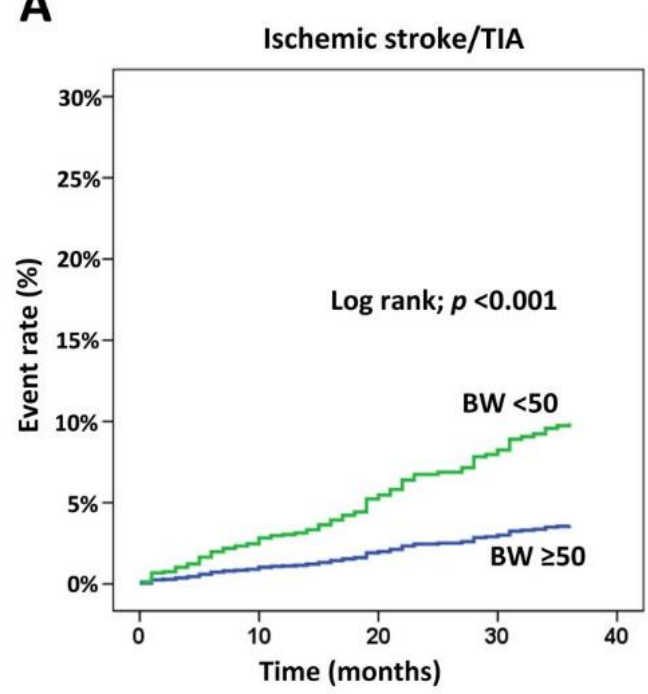

C

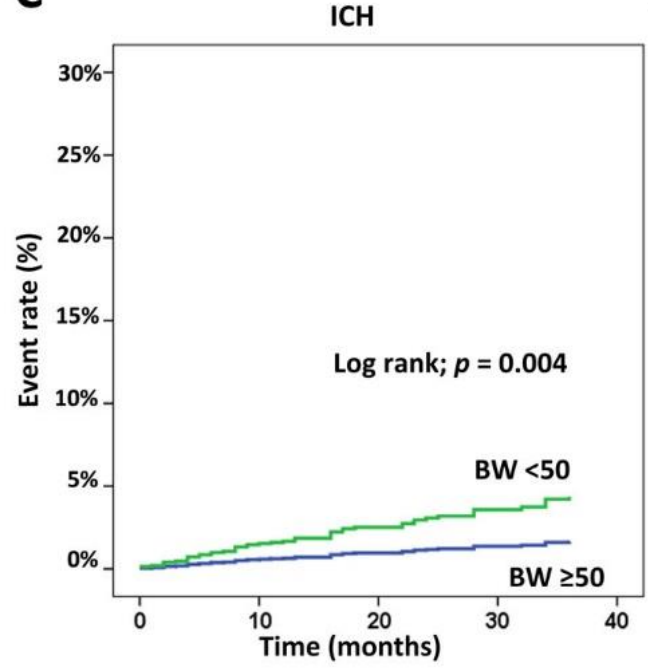

B

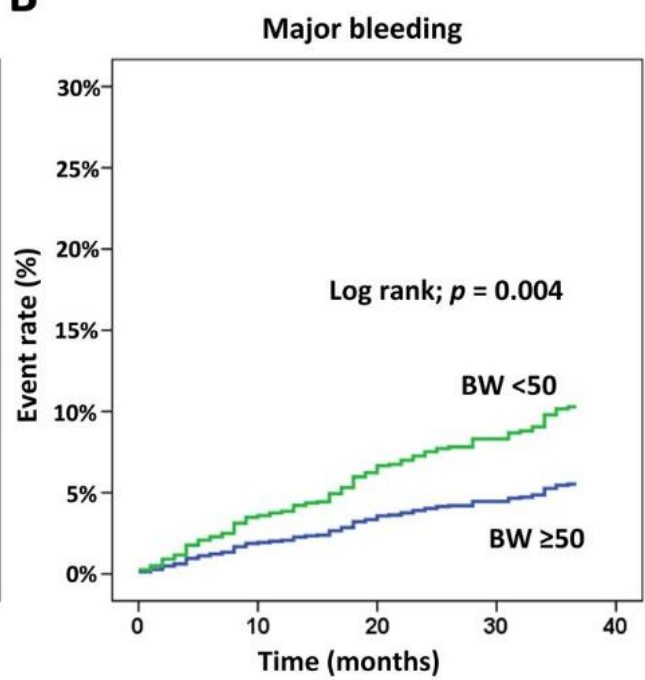

D

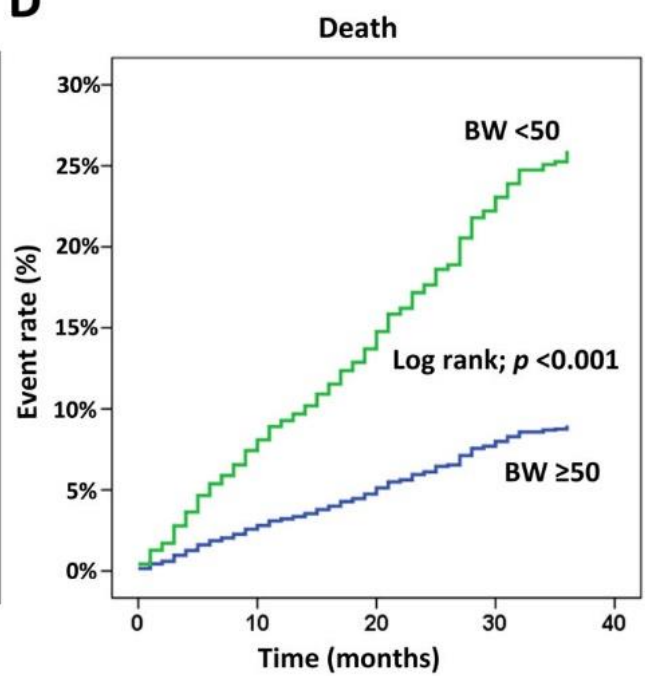

Figure 1. Cumulative event rate of ischemic stroke (IS)/transient ischemic attack (TIA) (A), major bleeding (B), intracerebral hemorrhage (ICH) (C), and death (D) compared between patients with body weight $(\mathrm{BW})<50$ and $\geq 50 \mathrm{~kg}$. A $p$-value $<0.05$ indicates statistical significance. Kaplan-Meier graph with log-rank test was used for analysis. BW, body weight; TIA, transient ischemic attack.

Regarding the cause of death, LBW had a higher rate of cardiovascular death (6.3\% vs. $2.1 \%$, $p<0.001)$. There was no significant difference in the rate of infection/sepsis and cancer as a cause of death between LBW and non-LBW group ( $2.9 \%$ vs. $1.8 \%, p=0.147$, for infection/sepsis, and $0.5 \%$ vs. $0.4 \%, p=0.683$, for cancer). 


\subsection{Subgroup Analyses}

Figure 2 shows the rates of ischemic stroke/transient ischemic attack, major bleeding, intracerebral hemorrhage, and death compared between body weight $<50$ and $\geq 50 \mathrm{~kg}$ among male patients, female patients, patients aged $\geq 65$ years, and patients aged $<65$ years. Patients with LBW were at significantly increased risk for all clinical outcomes among all patients as well as in each subgroup, except among those aged $<65$ years. The small number of patients in the latter age subgroup may have limited any statistically significant association. Females had a significantly higher rate of ischemic stroke than males, whereas males had a significantly higher rate of major bleeding compared to females. The older adult population had a significantly higher rate of all clinical events compared to the younger adult group.
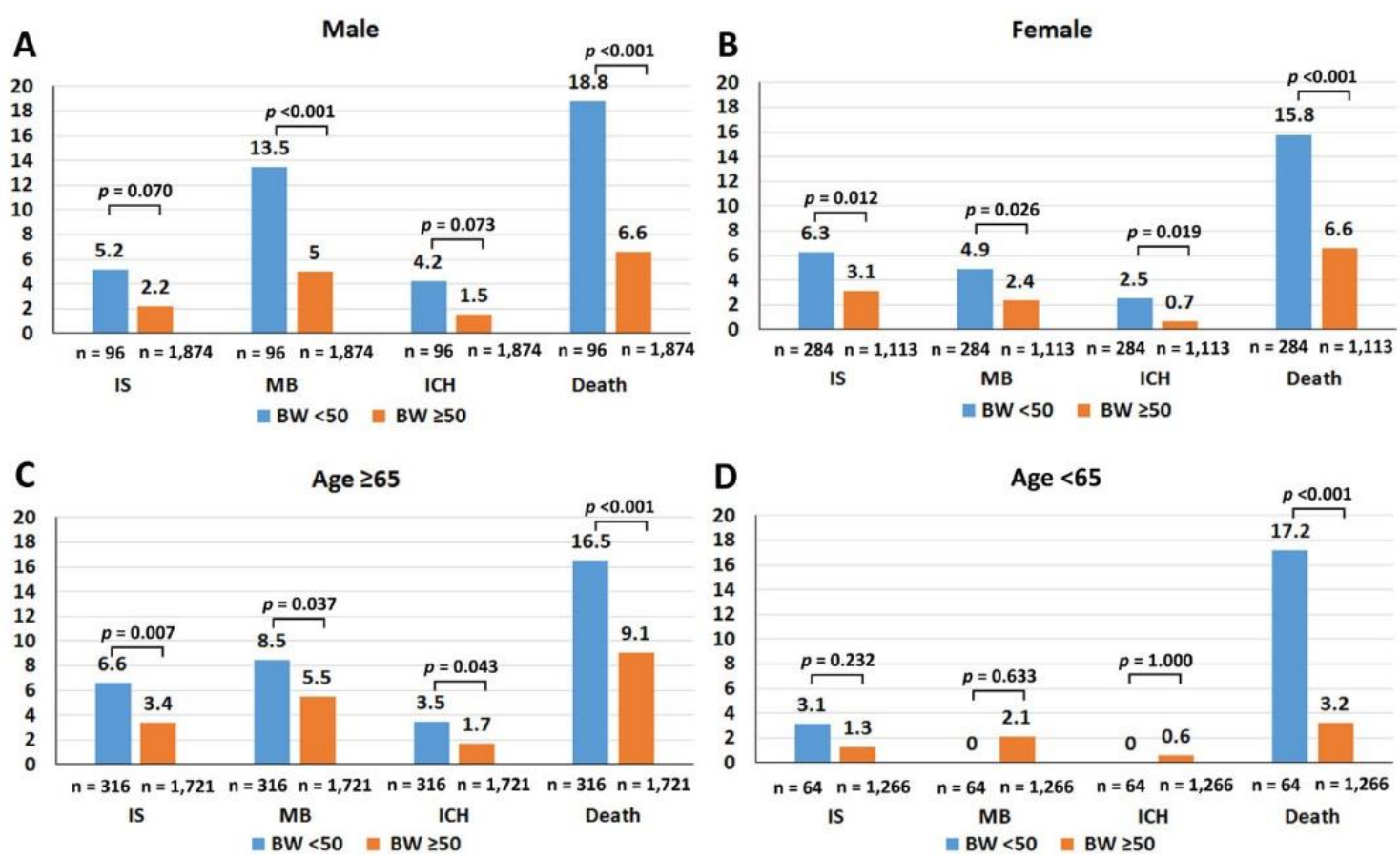

Figure 2. Rates of ischemic stroke (IS)/transient ischemic attack (TIA), major bleeding (MB), intracerebral hemorrhage (ICH), and death compared between body weight (BW) $<50$ and $\geq 50 \mathrm{~kg}$ among male patients (A), female patients (B), patients aged $\geq 65$ years (C), and patients aged $<65$ years (D). The chi-square test was used for analysis. A $p$-value $<0.05$ indicates statistical significance.

The rate of clinical outcomes among patients with body weight $<50$ and $\geq 50 \mathrm{~kg}$ is shown in Table 2. Ischemic stroke, major bleeding, and ICH were all significantly increased when body weight was below $50 \mathrm{~kg}$.

Table 2. Rates of clinical outcomes compared among different body weight groups.

\begin{tabular}{ccccc}
\hline & $\begin{array}{c}\text { All } \\
(\boldsymbol{n}=\mathbf{3 3 6 7 )}\end{array}$ & $\begin{array}{c}\text { BW }<\mathbf{5 0} \\
(\boldsymbol{n}=\mathbf{3 8 0})\end{array}$ & $\begin{array}{c}\text { BW } \geq \mathbf{5 0} \\
(\boldsymbol{n}=\mathbf{2 9 8 7})\end{array}$ & $\boldsymbol{p}$-Value \\
\hline Ischemic stroke/TIA & $99(2.9 \%)$ & $23(6.1 \%)$ & $76(2.5 \%)$ & $<0.001$ \\
Major bleeding & $148(4.4 \%)$ & $27(7.1 \%)$ & $121(4.1 \%)$ & 0.006 \\
ICH & $48(1.4 \%)$ & $11(2.9 \%)$ & $37(1.2 \%)$ & 0.010 \\
Death & $260(7.7 \%)$ & $63(16.6 \%)$ & $197(6.6 \%)$ & $<0.001$ \\
$\begin{array}{c}\text { Ischemic stroke/TIA or major } \\
\text { bleeding or death }\end{array}$ & $414(12.3 \%)$ & $90(23.7 \%)$ & $324(10.8 \%)$ & $<0.001$ \\
\hline
\end{tabular}

Data presented as number and percentage; A $p$-value $<0.05$ indicates statistical significance. Abbreviations: $\mathrm{BW}$, body weight; TIA, transient ischemic attack; ICH, intracerebral hemorrhage. 


\subsection{Multivariate Analysis}

Associations between adverse clinical outcomes and low body weight alone and low body weight adjusted for different factors are shown as a Forest plot in Figure 3. LBW was independently associated with increased risk of all main clinical outcomes, including ischemic stroke, major bleeding, ICH, and death. LBW remained independently associated with all four of these clinical outcomes after adjustment for all three different sets of potential confounders.

Hazard ratio $(95 \% \mathrm{Cl})$

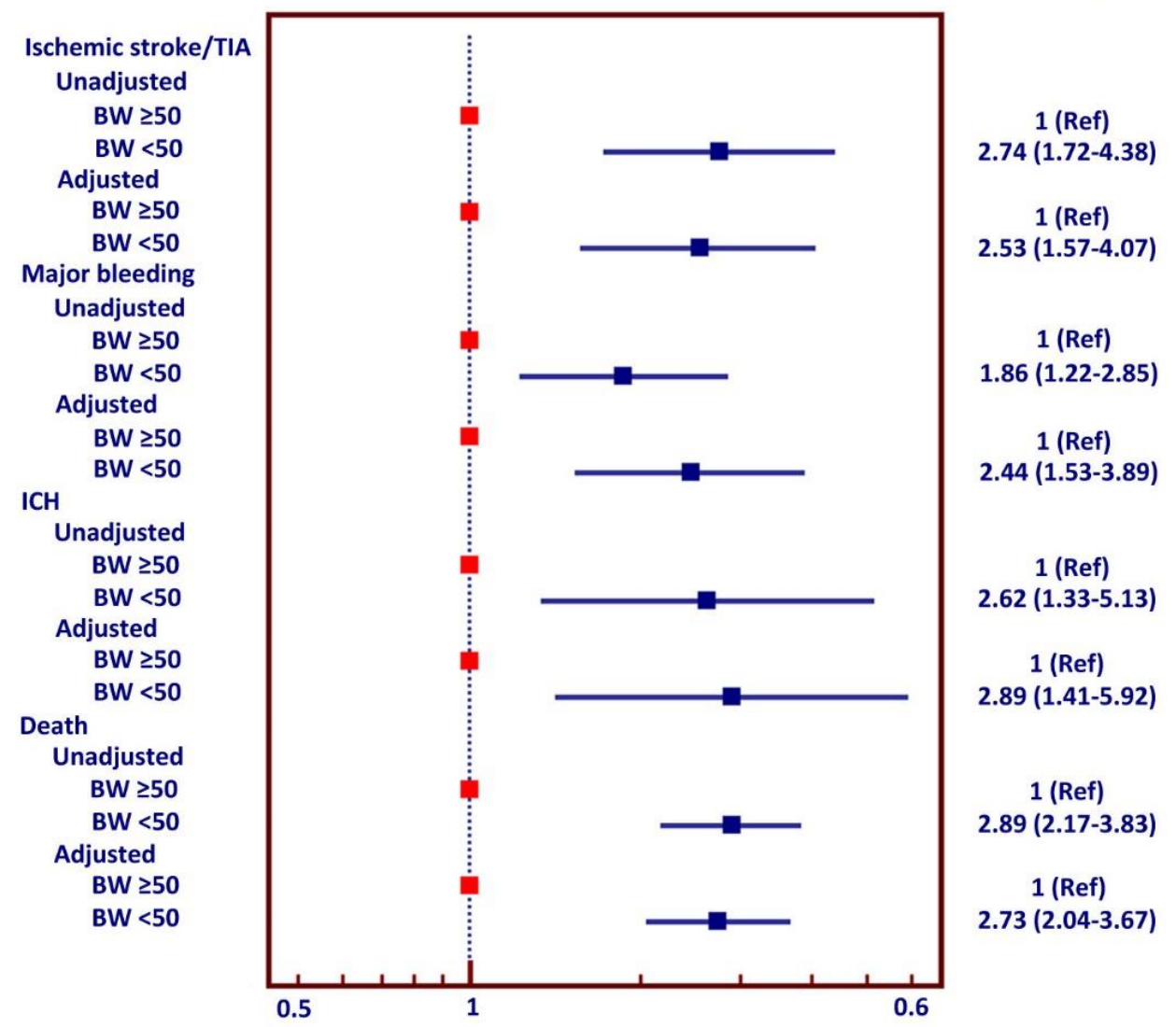

Figure 3. Forest plot shows unadjusted and adjusted hazard ratio of ischemic stroke/transient ischemic attack (TIA), major bleeding, intracerebral hemorrhage (ICH), and death in patients with low bodyweight and non-low body weight. The Cox proportional hazards model was used for analysis.

\subsection{Body Weight as a Continuous Variable}

Cox models with restricted cubic splines by treating body weight as continuous data demonstrated that LBW increased the risk of ischemic stroke/TIA, major bleeding, ICH, and death (Figure 4). The risk of all clinical events increased when body weight was below $60 \mathrm{~kg}$ and markedly increased when the body weight was below $50 \mathrm{~kg}$. 

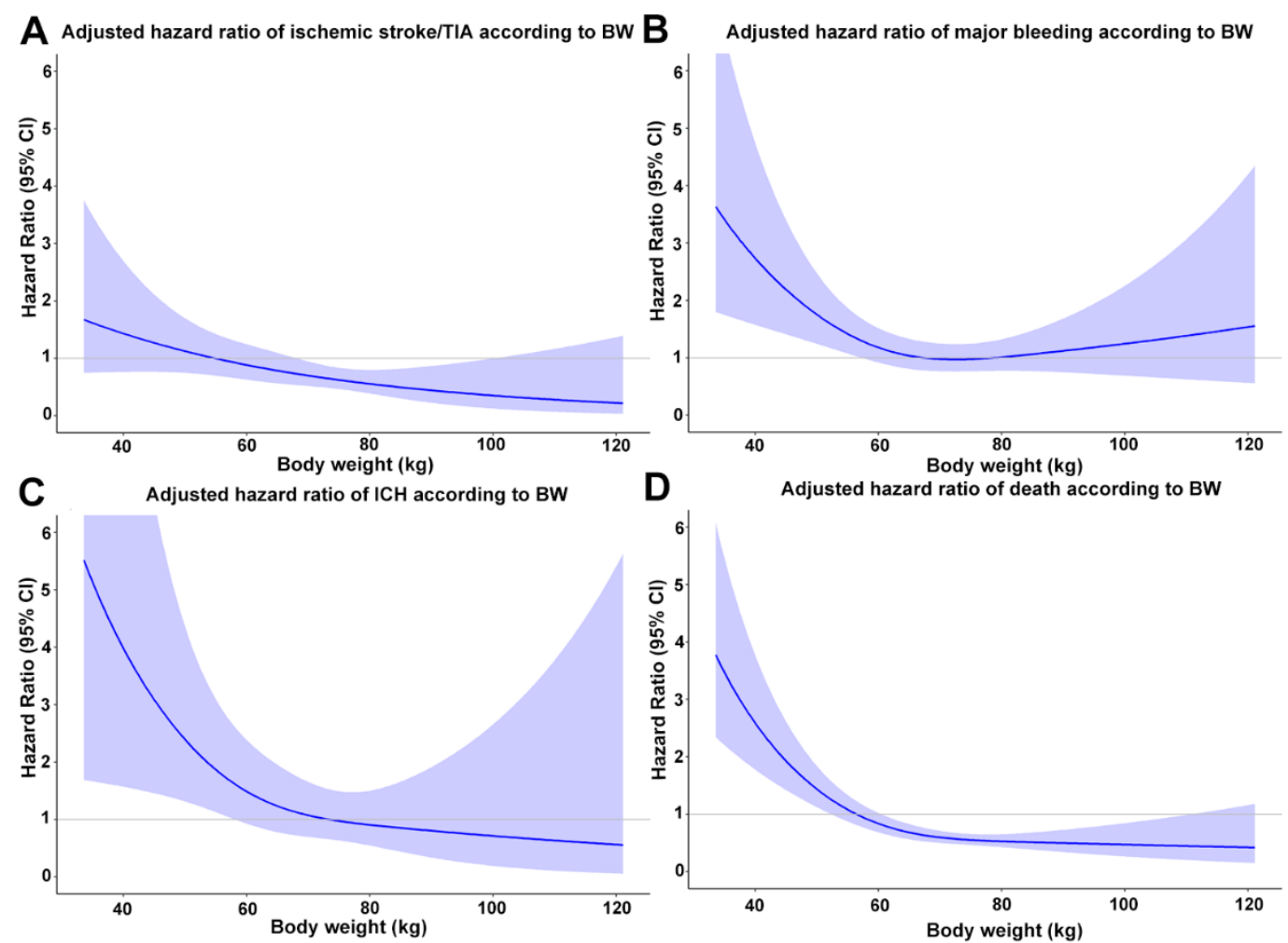

Figure 4. Restricted cubic splines graph showing Hazard ratio $(95 \% \mathrm{CI})$ of ischemic stroke/transient ischemic attack (TIA) (A), major bleeding (B), intracerebral hemorrhage (ICH) (C), and death (D) in patients with different body weight adjusted for age, sex, and comorbid conditions (coronary artery disease, heart failure, smoking status, diabetes, hypercholesterolemia, hypertension, history of ischemic stroke, history of major bleeding, renal replacement therapy, cardiac implantable electronic devices). Cox regression models with restricted cubic splines were used for analysis. CI, confidence interval.

\subsection{Sensitivity Analysis}

We performed additional analysis for low body mass index (BMI) $\left(<18.5 \mathrm{~kg} / \mathrm{m}^{2}\right)$ which is considered underweight by Asia-Pacific criteria [18] as compared to other BMI groups for measurement outcomes. We demonstrated that the underweight group had a higher rate of ischemic stroke/TIA, major bleeding, $\mathrm{ICH}$, and death compared to the non-underweight group, with $p$ values of $0.003,0.007$, 0.024 , and $<0.001$, respectively.

During the average follow-up duration of $25.7 \pm 10.6$ months, there were some changes in risk factors and medications that might have influence on the outcomes. The rate of new development of specific risk factors during follow-up were $4.75 \%$ for heart failure, $6.37 \%$ for hypertension, $8.94 \%$ for age $\geq 75$ years, $3.63 \%$ for diabetes, $3.04 \%$ for prior stroke or TIA, $1.04 \%$ for vascular disease, and $8.63 \%$ for age $65-74$ years. OAC and antiplatelet were introduced during follow-up in $30.65 \%$ and $5.31 \%$, whereas the discontinuation rate was $0.47 \%$ and $6.69 \%$, respectively. The Generalized Estimating Equation (GEE) with exchangeable correlation structure was used to analyse the effect of LBW on clinical outcomes, with the adjustment of time varying covariates. The results of the analysis demonstrated that LBW remained an independent predictor for ischemic stroke/TIA, major bleeding, and ICH with the adjusted Odds ratio of $2.18(1.27-3.74), p=0005 ; 2.07(1.26-3.39), p=0.004$; and 3.15 (1.47-6.77), $p=0.003$, respectively.

Although we tried to adjust for potential confounders, there might be a concern whether the effect of LBW on clinical outcomes is due to a difference in baseline characteristics between the two groups such as CHA2DS2-VASc score, gender, and age. We, therefore, selected only patients with CHA2DS2-VASc score $\geq 2$ and ran the analysis for the effect of LBW on clinical outcomes. The results 
showed that LBW remained a significant predictor for clinical outcomes, with the adjusted HR for LBW for ischemic stroke/TIA, major bleeding, ICH, and death being 2.64 (1.63-4.27), $p<0.001 ; 2.47$ (1.56-3.91), $p<0.001 ; 3.19$ (1.55-6.58), $p=0.002$; and 2.47 (1.83-3.35), $p<0.001$, respectively.

Regarding the influence of gender and age on the results of LBW on clinical outcomes, we used an interaction test. The results demonstrated that there was no significant interaction of gender or age group on the effect of LBW on clinical outcomes. The interaction $p$-values of gender were $0.637,0.406$, 0.734 , and 0.279 for ischemic stroke/TIA, major bleeding, ICH, and death, respectively. The interaction $p$-values of the elderly ( $\geq 65$ years) and non-elderly ( $<65$ years) were $0.337,0.729,0.257$, and 0.297 for the respective outcomes. Figure S2 in Supplementary Materials shows a restricted cubic splines graph of LBW and ischemic stroke/TIA and major bleeding stratified by gender (A and B) and age group (C and D).

To determine whether the body weight cut off is different for males and females, we ran a restricted cubic splines graph of ischemic stroke/TIA and major bleeding stratified by gender (Figure S2A,B in Supplementary Material) using body weight as a continuous variable. We demonstrated that the adjusted hazard ratio of ischemic stroke/TIA started rising at the body weight below $60 \mathrm{~kg}$ in both genders and significantly increased when body weight was below $50 \mathrm{~kg}$. For major bleeding, the adjusted hazard ratio for males started rising at body weight below $65 \mathrm{~kg}$ and significantly increased at body weight below $50 \mathrm{~kg}$. For females, the adjusted hazard ratio was increased at body weight below $50 \mathrm{~kg}$.

For concern whether the reference group should be relatively normal weight individuals, we performed additional analysis. Since our cut off for LBW was approximately 10 percentiles, we ran an analysis for the effect of LBW on clinical outcomes by excluding patients with body weight above the 90th percentile. The adjusted hazard ratios of LBW compared to the reference group were 2.40 (1.49-3.88), $p<0.001 ; 2.25$ (1.42-3.55), $p=0.001 ; 2.85$ (1.39-5.86), $p=0.004$; and 2.74 (2.04-3.69), $p<0.001$ for ischemic stroke/TIA, major bleeding, ICH, and death, respectively.

\subsection{Effects of $O A C$ on Clinical Outcomes}

Additional analyses were performed to evaluate the effect of OAC use on the rate of clinical outcomes among patients with different body weights (Figure 5A). In patients with LBW, OAC use was not associated with lowered ischemic stroke/TIA but an increased risk of major bleeding. OAC use increased the risk of ICH greater than 3-fold compared to those not on OAC. The risk of ICH also increased approximately 3 -fold in patients with body weight $\geq 50 \mathrm{~kg}$ compared to those not on OAC (3.4\% vs. $1.1 \%$ ). The risk of ICH in patients on OAC with body weight $<50 \mathrm{~kg}$ was more than two-fold compared to those with body weight $\geq 50 \mathrm{~kg}(3.4 \%$ vs. $1.5 \%)$. The interaction $p$-values of OAC use for the effect of body weight on ischemic stroke/TIA, major bleeding, ICH, and death were $0.025,0.871$, 0.981 , and 0.954 , respectively. For those who were on warfarin, average time in therapeutic range (TTR) was $53.5 \pm 26.4 \%$. TTR was $53.0 \pm 26.2 \%$ in patients with low BW compared to $53.6 \pm 26.4 \%$ for those without low BW $(p=0.722)$.

\subsection{Net Clinical Benefit}

NCB was calculated for all patients and for high-risk patients defined as men with CHA2DS2-VASc score more than 1 or women with CHA2DS2-VASc score more than 2. When weighting ICH as 1.5 as mentioned earlier, NCB (95\% CI) of OAC compared to no OAC was $-0.56(-0.73$ to -0.38$)$ per 100 person-years, which means that OAC may have a higher risk than benefit. NCB was more negative (more risk than benefit) in patients with LBW $(-2.35(-2.88$ to -1.84$))$ per 100 person-years] compared to non-LBW ( -0.34 (-0.53 to -0.15$)$ ) per 100 person-years] group (Figure $5 \mathrm{~B})$. Warfarin was used in $2310(91.1 \%)$ of patients who are on OAC. Our study had limited power to compare outcomes of warfarin and NOACs due to the small number of NOACs, especially in the LBW group. However, the results of NCB showed that NOACs had NCB of 1.35 (1.17 to 1.52) per 100 person-years compared to warfarin, which means that NOAC provided more benefit than risk compared to warfarin. NCB of 
NOACs compared to warfarin in LBW and non-LBW were 3.48 ( -0.21 to 7.23$)$ and 1.05 (0.73 to 1.37$)$ per 100 person-years, respectively, which means that the benefit of NOACs over warfarin was more in the LBW group. Results of NCB in high-risk patients was in a similar direction for all patients.
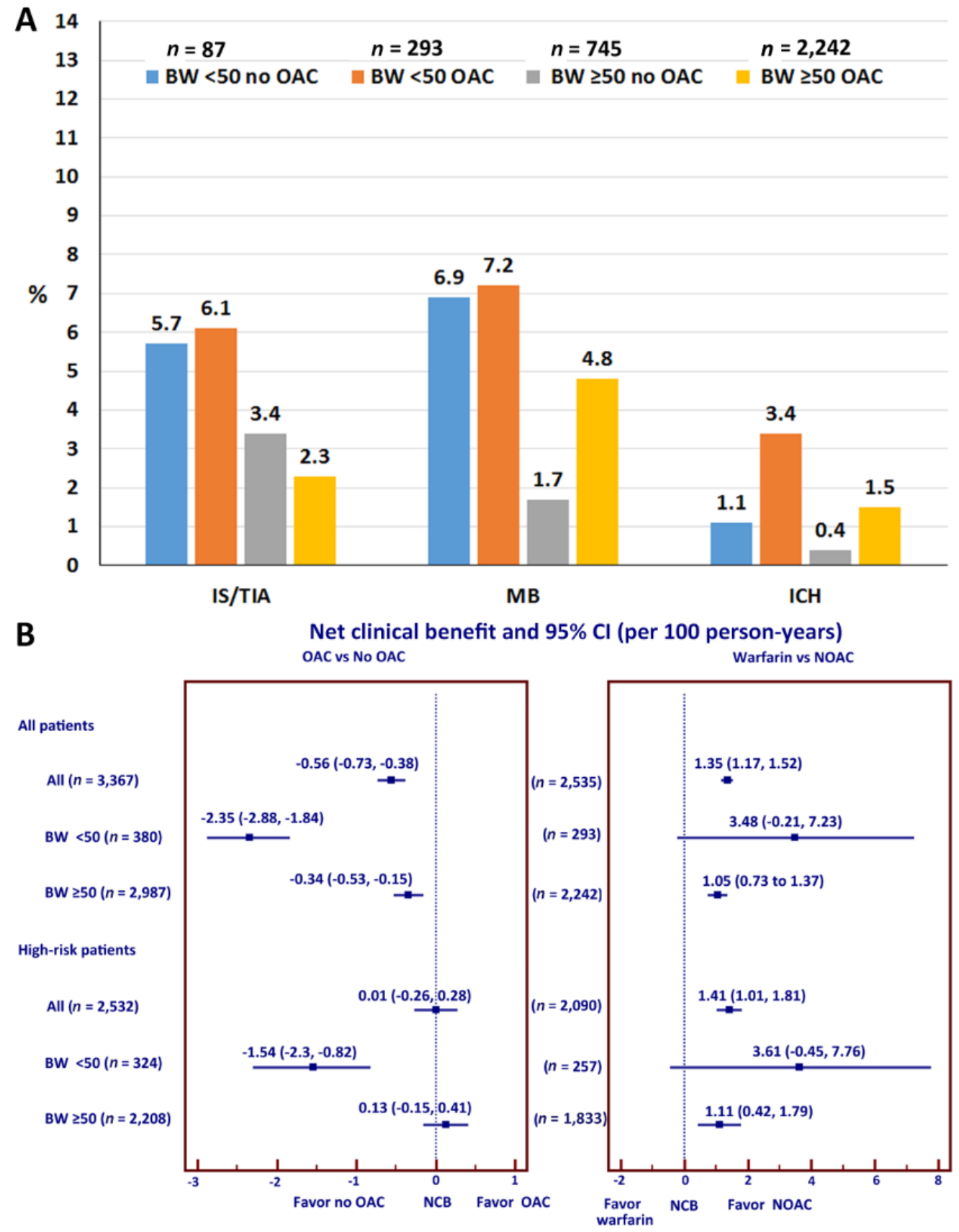

Figure 5. Oral anticoagulants and clinical outcomes. (A). Rates of ischemic stroke (IS)/transient ischemic attack (TIA), major bleeding (MB), and intracerebral hemorrhage (ICH) stratified by body weight (BW) and oral anticoagulation (OAC). The interaction $p$-values of OAC use for the effect of body weight on ischemic stroke/TIA, major bleeding, and ICH were $0.025,0.871,0.981$, respectively. (B). Net clinical benefit (NCB) of OAC compared to no OAC (left) and warfarin compared to non-vitamin $\mathrm{K}$ antagonist oral anticoagulant (NOAC) (right). Forest plot and Cox proportional hazards model was used for analysis. 


\section{Discussion}

In this prospective nationwide cohort of Thai patients with atrial fibrillation (AF), we show that LBW was present in $11.3 \%$ and was an independent predictor of increased risk of ischemic stroke, major bleeding, ICH, and death irrespective of OAC therapy. This is the largest contemporary cohort of Thai patients with NVAF.

Concerns whether LBW status increases the chance of developing a bleeding complication from OAC treatment has been reported [19]. A smaller body size means a smaller volume of distribution and thus, a regular dose of medication might increase the blood concentration of that medication to a level higher than would be achieved in someone with a normal body size [13]. This is particularly important for OAC medications, which may cause bleeding at inappropriately high blood concentrations. Certain OAC medications, such as apixaban and edoxaban, are recommended for consideration of a low-dose regimen in LBW patients [14].

Asians have a smaller average body size than Caucasians and there are concerns that Asian patients may be at higher risk for developing adverse effects from medications compared to larger body size counterparts [20]. For example, many anticoagulant drugs have a "Japanese dose" that is lower than the usual recommended dose [21]. Other studies have reported that the Asian population is more likely to have bleeding complications than white Europeans [7,22]. Indeed, warfarin-related ICH complications in NVAF patients are 4 times higher in Asian population than in Caucasian populations [7]. The recent NOAC trials have also showed that the Asian subgroup had higher rates of both major bleeding and ICH when on warfarin therapy [22]. This could be one of the reasons why a low proportion of the Asian population received OAC compared to Western populations [23] and even when they did receive warfarin, the proportion that had an INR within the target range was lower than that of patients in other countries [23].

The prevalence of NVAF in elderly Thai participants was $1.9 \%$ [24]. Only $40 \%$ of known AF subjects received warfarin while $60 \%$ received antiplatelets. The prevalence of underweight people among the Thai population was approximately $8.7 \%$ [25]. From Thailand national data on 522,699 patients with acute stroke, NVAF is one of the major factors leading to stroke [26].

Some previous studies reported that LBW increased the risk of adverse events in patients with NVAF [11,12]. Data from the Fushimi AF Registry showed that LBW significantly increased the risk of ischemic stroke among 2945 NVAF patients (HR: 2.19, 95\% CI: 1.57-3.04), but the risk of bleeding was comparable between the LBW and normal body weight groups (HR: 1.05, 95\% CI: 0.64-1.68). However, only about $50 \%$ of patients in the Fushimi AF Registry received OAC therapy [12]. In contrast, OAC was given to $75.3 \%$ of patients in our registry. The Fushimi AF Registry also showed the risk of death to be almost 3 times higher in the LBW group compared to the non-LBW group [12]. A study from the Korean National Health Insurance Database also found that patients with a body weight $<50 \mathrm{~kg}$ had a higher incidence of ischemic stroke, bleeding, and death compared to those with a body weight ranging from 50 to $60 \mathrm{~kg}$ [27]. These results are consistent with our findings (Table 2) that the risk of both ischemic stroke and bleeding was significantly increased when body weight was below $50 \mathrm{~kg}$. It should be noted that $100 \%$ of patients in the Korean study were on OAC and NOAC was used in $65 \%$ of their patients. In contrast, NOAC was used in only $9 \%$ of patients in our study.

A systematic review and meta-analysis compared data from randomized clinical trials of NOACs in patients with NVAF or venous thromboembolism (VTE) and showed that LBW increased the risk of ischemic stroke, but the rate of major bleeding was comparable between the LBW and non-LBW groups [11]. That group also compared obese versus non-obese patients and found that obese patients had a lower rate of ischemic stroke compared to non-obese patients, but the rate of major bleeding was not significantly different between the two weight groups [11]. Those findings are consistent with our observations that the rate of ischemic stroke was lower in patients with body weight $\geq 60 \mathrm{~kg}$ compared to $<60 \mathrm{~kg}$ and the rate markedly increased when body weight $<50 \mathrm{~kg}$. However, their finding of Boonyawat et al. [11] of a comparable bleeding event rate between the LBW and non-LBW groups is in contrast to our results. One possible explanation could be the weight cutoff used in the included 
studies. More specifically, half of the studies they included used $50 \mathrm{~kg}$ as the cutoff and the other studies used $60 \mathrm{~kg}$ as the cutoff. Once again, we found the rates of both ischemic stroke and bleeding to be significantly increased when body weight was below $50 \mathrm{~kg}$. The increased risk of major bleeding in the LBW group of our study cannot be explained by the rate of OAC use, there was no significant difference in the 2 groups (77.1\% for LBW and 75.1\% for non-LBW group, $p=0.384$ ), and it cannot be explained by the rate of OAC plus antiplatelet, which was more common in the non-LBW group $(4.2 \%$ for LBW and $9.8 \%$ for non-LBW group, $p<0.001$ ). The use of NOAC may be the solution for ischemic stroke and major bleeding risk reduction. Our study also demonstrated that the NCB results favored NOAC over warfarin for both the LBW and non-LBW group. The NCB of NOAC in the LBW group was greater than the non-LBW group. Recent meta-analysis demonstrated that use of NOAC contributed to the reduction of both ischemic stroke and major bleeding across all groups of body weight [28]. The results of our study did not have enough power to analyze the effect of NOAC on clinical outcomes due to the limited number of patients using NOAC. However, data from our study showed that compared to warfarin, the rate of major bleeding was significantly lower with NOAC (5.4\% for warfarin, $2.2 \%$ for NOAC, $p=0.040)$ and the rate of ischemic stroke/TIA was numerically lower for NOAC $(2.9 \%$ for warfarin, $1.3 \%$ for NOAC, $p=0.180$ ). A slightly higher time in the therapeutic range in the LBW group in our study should not explain the increased risk of adverse outcomes in the LBW group since the difference is small, the average time in the therapeutic range is similar, and the LBW group also had increased risk of ischemic stroke/TIA, not just major bleeding.

We use $50 \mathrm{~kg}$ as the cut off criteria for low body weight due to the following reasons: (1) weight is a simple measure and it is easier to use than BMI, which needs a calculation. For example, one of the dose reduction criteria of apixaban is body weight less than $60 \mathrm{~kg}$ (2) we use less than $50 \mathrm{~kg}$ to compare the results with previous publications [11,12]. We performed additional analysis comparing BMI using the cut off of $18.5 \mathrm{~kg} / \mathrm{m}^{2}$, which is considered underweight by Asia-Pacific population criteria [18], and normal weight $\left(18.5-22.9 \mathrm{~kg} / \mathrm{m}^{2}\right)$, overweight $\left(23-24.9 \mathrm{~kg} / \mathrm{m}^{2}\right)$, and obesity $\left(\geq 25 \mathrm{~kg} / \mathrm{m}^{2}\right)$. Patients in the underweight group had a higher rate of a composite of ischemic stroke/TIA, major bleeding, and death compared to the other three groups ( $23.7 \%$ vs. $17.2 \%$ vs. $10.3 \%$ vs. $9.3 \%$, respectively). The rate of individual clinical outcomes was also higher in the underweight group defined by the BMI.

Several mechanisms for the linkage between LBW and increased risk of ischemic stroke and bleeding have been proposed. First, LBW may be associated with an altered pharmacokinetic effect of the anticoagulant, which increases bleeding risk [13]. Second, these increased risks could be related to genetic factors that are also associated with smaller body size [20]. Third, LBW patients may have a lower lean body mass, which was reported to be associated with a worse prognosis for cardiovascular outcome [29]. Fourth, obese patients tended to adhere to guideline recommended therapy more closely than their lower body weight counterparts [30]. Fifth, obese patients tend to have an increased level of cardioprotective adipokines [31,32] and LBW is associated with impaired endothelium-dependent vasodilatation [33]. Sixth, patients with LBW may have a different neuro-hormonal response to exercise [34]. In addition to LBW being shown to increase the risk of cardiovascular events in patients at high risk for atherothrombotic events [35], levels of inflammatory markers are also increased in patients with LBW [36,37]. Lastly, people from many Asian countries had a less favorable body composition and adipokines. Although they might have a lesser lean body mass, many of them had a greater visceral fat component, had lower adiponectin levels, and higher resistin levels, creating a metabolically abnormal normal weight phenotype with unfavorable metabolic health, even at normal weight or underweight [38].

\section{Limitations}

This study has some limitations. First, the results of this study may not be generalizable to Thai patients in other care settings or to patients that are not Thai. The patients in this registry were mainly enrolled from large hospitals and most received their care from cardiologists. In contrast, patients that receive care in less sophisticated settings are often treated by internists or general practitioners. 
Second, OAC in our study was mainly warfarin, which may limit generalizability to populations with a large proportion of NOAC usages. Besides, we did not have data on the dose of NOACs that might have an effect on the clinical outcomes. The strength of this study is its prospective design, the large size of the study population, and the fact that the data were collected from 27 large hospitals located all across Thailand.

\section{Conclusions}

LBW was independently associated with increased risk of ischemic stroke, major bleeding, ICH, and death in Thai patients with NVAF. OAC may not have benefit in a LBW group. This should be carefully considered when balancing the risks and benefits of stroke prevention among patients with different body weights.

Supplementary Materials: The following are available online at http://www.mdpi.com/2077-0383/9/9/2713/s1, Figure S1: Distribution of body weight among male patients (A), female patients (B), patients aged $\geq 65$ years (C), and patients aged < 65 years (D); Figure S2: Restricted cubic splines graph showing Hazard ratio $(95 \% \mathrm{CI})$ stratified by gender ( $A$ and $B$ ) and age group (C and D) for ischemic stroke/transient ischemic attack (TIA) (A, C) and major bleeding $(\mathrm{B}, \mathrm{D})$ in patients with different body weight adjusted for age, sex, and comorbid conditions (coronary artery disease, heart failure, smoking status, diabetes, hypercholesterolemia, hypertension, history of ischemic stroke, history of major bleeding, renal replacement therapy, cardiac implantable electronic devices). Cox regression models with restricted cubic splines was used for analysis.

Author Contributions: R.K.-concept and design, data acquisition, interpretation of data, manuscript preparation, manuscript revision, and manuscript review; P.C. and S.K.- data acquisition, manuscript revision, and manuscript review; C.K. and A.Y.-data analysis, manuscript revision, and manuscript review; and, G.Y.H.L.-concept and design, data acquisition, manuscript revision, and manuscript review. All authors have read and agreed to the published version of the manuscript.

Funding: This study was funded by grants from the Health Systems Research Institute (HSRI) (grant no. 59-053), the Heart Association of Thailand under the Royal Patronage of H.M., the King. None of the aforementioned funding sources influenced any aspect of this study or the decision of the authors to submit this manuscript for publication.

Acknowledgments: The authors gratefully acknowledge Pontawee Kaewcomdee and Olaree Chaiphet for data management and nurse coordinators for their assistance with patient enrollment and their commitment to maintaining a high level of data quality. The authors would also like to thank all of the patients that agreed to participate in this study. List of COOL-AF investigators: Buddhachinaraj Hospital: Tomorn Thongsri, Central Chest Institute of Thailand: Kriengkrai Hengrussamee, Charoen Krung Pracha Rak Hospital: Pattraporn Srirattana, Chiangrai Prachanukroh Hospital: Wattana Wongtheptien, Chonburi Hospital: Pornchai Ngamjanyaporn, Faculty of Medicine, Chiang Mai University: Arintaya Phrommintikul, Faculty of Medicine, Chulalongkorn University: Smonporn Boonyaratavej, Faculty of Medicine, Naresuan University: Pongpun Jittham, Faculty of Medicine, Prince of Songkla University: Treechada Wisartpong, Faculty of Medicine Ramathibodi Hospital, Mahidol University: Sirin Apiyasawat, Faculty of Medicine Siriraj Hospital, Mahidol University: Arjbordin Winijkul, and Rungroj Krittayaphong, Faculty of Medicine, Thammasat University (Rangsit Campus): Roj Rojretamphai, Faculty of Medicine Vajira Hospital, Navamindradhiraj University: Kulyot Jongpiputvanich, Golden Jubilee Medical Center: Somchai Dutsadeevettakul, Khon Kaen Hospital: Chaisit Wongwipaporn, Lampang Hospital: Tanita Bunyapipat, Nakorn Ratchasima Hospital: Weerapan Wiwatworapan, Nakornping Hospital: Khanchai Siriwattana, Phramongkutklao College of Medicine: Tharanit Chantrarat, Police General Hospital: Kasem Rattanasumawong, Prapokklao Hospital (Chanthaburi): Wiwat Kanjanarutjawiwat, Queen Savang Vadhana Memorial Hospital: Sakaorat Kornbongkotmas; Ratchaburi Hospital: Thanasak Patmuk, Sapphasitthiprasong Hospital: Praprut Thanakitcharu, Surat Thani Hospital: Suchart Arunsiriwattana, Surin Hospital: Thaworn Choochunklin; Udon Thani Hospital: Sumon Tangsuntornwiwat.

Conflicts of Interest: GYHL: Consultant for Bayer/Janssen, BMS/Pfizer, Medtronic, Boehringer Ingelheim, Novartis, Verseon, and Daiichi-Sankyo. Speaker for Bayer, BMS/Pfizer, Medtronic, Boehringer Ingelheim, and Daiichi-Sankyo. No fees are directly received personally. Other authors hereby declare no personal or professional conflicts of interest relating to any aspect of this particular study. The funders had no role in the design of the study; in the collection, analyses, or interpretation of data; in the writing of the manuscript, or in the decision to publish the results.

\section{References}

1. Lip, G.; Freedman, B.; De Caterina, R.; Potpara, T.S. Stroke prevention in atrial fibrillation: Past, present and future. Comparing the guidelines and practical decision-making. Thromb. Haemost. 2017, 117, 1230-1239. [CrossRef] [PubMed] 
2. Kirchhof, P.; Benussi, S.; Kotecha, D.; Ahlsson, A.; Atar, D.; Casadei, B.; Castella, M.; Diener, H.C.; Heidbuchel, H.; Hendriks, J.; et al. 2016 ESC Guidelines for the management of atrial fibrillation developed in collaboration with EACTS. Eur. Heart J. 2016, 37, 2893-2962. [CrossRef] [PubMed]

3. January, C.T.; Wann, L.S.; Calkins, H.; Chen, L.Y.; Cigarroa, J.E.; Cleveland, J.C., Jr.; Ellinor, P.T.; Ezekowitz, M.D.; Field, M.E.; Furie, K.L.; et al. 2019 AHA/ACC/HRS Focused Update of the 2014 AHA/ACC/HRS Guideline for the Management of Patients with Atrial Fibrillation: A Report of the American College of Cardiology/American Heart Association Task Force on Clinical Practice Guidelines and the Heart Rhythm Society. J. Am. Coll. Cardiol. 2019. [CrossRef]

4. Lip, G.Y.H.; Banerjee, A.; Boriani, G.; Chiang, C.E.; Fargo, R.; Freedman, B.; Lane, D.A.; Ruff, C.T.; Turakhia, M.; Werring, D.; et al. Antithrombotic Therapy for Atrial Fibrillation: CHEST Guideline and Expert Panel Report. Chest 2018, 154, 1121-1201. [CrossRef]

5. Healey, J.S.; Oldgren, J.; Ezekowitz, M.; Zhu, J.; Pais, P.; Wang, J.; Commerford, P.; Jansky, P.; Avezum, A.; Sigamani, A.; et al. Occurrence of death and stroke in patients in 47 countries 1 year after presenting with atrial fibrillation: A cohort study. Lancet 2016, 388, 1161-1169. [CrossRef]

6. Tse, H.F.; Wang, Y.J.; Ahmed Ai-Abdullah, M.; Pizarro-Borromeo, A.B.; Chiang, C.E.; Krittayaphong, R.; Singh, B.; Vora, A.; Wang, C.X.; Zubaid, M.; et al. Stroke prevention in atrial fibrillation-An Asian stroke perspective. Heart Rhythm 2013, 10, 1082-1088. [CrossRef]

7. Shen, A.Y.; Yao, J.F.; Brar, S.S.; Jorgensen, M.B.; Chen, W. Racial/ethnic differences in the risk of intracranial hemorrhage among patients with atrial fibrillation. J. Am. Coll. Cardiol. 2007, 50, 309-315. [CrossRef]

8. Chao, T.F.; Liu, C.J.; Tuan, T.C.; Chen, S.J.; Wang, K.L.; Lin, Y.J.; Chang, S.L.; Lo, L.W.; Hu, Y.F.; Chen, T.J.; et al. Comparisons of CHADS2 and CHA2DS2-VASc scores for stroke risk stratification in atrial fibrillation: Which scoring system should be used for Asians? Heart Rhythm 2016, 13, 46-53. [CrossRef]

9. Chao, T.F.; Lip, G.Y.; Liu, C.J.; Tuan, T.C.; Chen, S.J.; Wang, K.L.; Lin, Y.J.; Chang, S.L.; Lo, L.W.; Hu, Y.F.; et al. Validation of a Modified CHA2DS2-VASc Score for Stroke Risk Stratification in Asian Patients With Atrial Fibrillation: A Nationwide Cohort Study. Stroke 2016, 47, 2462-2469. [CrossRef]

10. Hamatani, Y.; Yamashita, Y.; Esato, M.; Chun, Y.H.; Tsuji, H.; Wada, H.; Hasegawa, K.; Abe, M.; Lip, G.Y.; Akao, M. Predictors for Stroke and Death in Non-Anticoagulated Asian Patients with Atrial Fibrillation: The Fushimi AF Registry. PLoS ONE 2015, 10, e0142394. [CrossRef]

11. Boonyawat, K.; Caron, F.; Li, A.; Chai-Adisaksopha, C.; Lim, W.; Iorio, A.; Lopes, R.D.; Garcia, D.; Crowther, M.A. Association of body weight with efficacy and safety outcomes in phase III randomized controlled trials of direct oral anticoagulants: A systematic review and meta-analysis. J. Thromb. Haemost. 2017, 15, 1322-1333. [CrossRef] [PubMed]

12. Hamatani, Y.; Ogawa, H.; Uozumi, R.; Iguchi, M.; Yamashita, Y.; Esato, M.; Chun, Y.H.; Tsuji, H.; Wada, H.; Hasegawa, K.; et al. Low Body Weight Is Associated With the Incidence of Stroke in Atrial Fibrillation Patients-Insight From the Fushimi AF Registry. Circ. J. 2015, 79, 1009-1017. [CrossRef] [PubMed]

13. Upreti, V.V.; Wang, J.; Barrett, Y.C.; Byon, W.; Boyd, R.A.; Pursley, J.; LaCreta, F.P.; Frost, C.E. Effect of extremes of body weight on the pharmacokinetics, pharmacodynamics, safety and tolerability of apixaban in healthy subjects. Br. J. Clin. Pharm. 2013, 76, 908-916. [CrossRef] [PubMed]

14. Granger, C.B.; Alexander, J.H.; McMurray, J.J.; Lopes, R.D.; Hylek, E.M.; Hanna, M.; Al-Khalidi, H.R.; Ansell, J.; Atar, D.; Avezum, A.; et al. Apixaban versus warfarin in patients with atrial fibrillation. N. Engl. J. Med. 2011, 365, 981-992. [CrossRef]

15. Krittayaphong, R.; Winijkul, A.; Methavigul, K.; Wongtheptien, W.; Wongvipaporn, C.; Wisaratapong, T.; Kunjara-Na-Ayudhya, R.; Boonyaratvej, S.; Komoltri, C.; Kaewcomdee, P.; et al. Risk profiles and pattern of antithrombotic use in patients with non-valvular atrial fibrillation in Thailand: A multicenter study. BMC Cardiovasc. Disord. 2018, 18, 174. [CrossRef]

16. Schulman, S.; Kearon, C.; Subcommittee on Control of Anticoagulation of the Scientific; Standardization Committee of the International Society on Thrombosis and Haemostasis. Definition of major bleeding in clinical investigations of antihemostatic medicinal products in non-surgical patients. J. Thromb. Haemost. 2005, 3, 692-694. [CrossRef]

17. Singer, D.E.; Chang, Y.; Fang, M.C.; Borowsky, L.H.; Pomernacki, N.K.; Udaltsova, N.; Go, A.S. The net clinical benefit of warfarin anticoagulation in atrial fibrillation. Ann. Intern. Med. 2009, 151, 297-305. [CrossRef] 
18. Pan, W.H.; Yeh, W.T. How to define obesity? Evidence-based multiple action points for public awareness, screening, and treatment: An extension of Asian-Pacific recommendations. Asia Pac. J. Clin. Nutr. 2008, 17, 370-374.

19. Pijl, H.; Meinders, A.E. Bodyweight change as an adverse effect of drug treatment. Mechanisms and management. Drug Saf. 1996, 14, 329-342. [CrossRef]

20. Eliasson, E. Ethnicity and adverse drug reactions. BMJ 2006, 332, 1163-1164. [CrossRef]

21. Hori, M.; Matsumoto, M.; Tanahashi, N.; Momomura, S.; Uchiyama, S.; Goto, S.; Izumi, T.; Koretsune, Y.; Kajikawa, M.; Kato, M.; et al. Rivaroxaban vs. warfarin in Japanese patients with atrial fibrillation-The J-ROCKET AF study. Circ. J. 2012, 76, 2104-2111. [CrossRef] [PubMed]

22. Lip, G.Y.; Wang, K.L.; Chiang, C.E. Non-vitamin K antagonist oral anticoagulants (NOACs) for stroke prevention in Asian patients with atrial fibrillation: Time for a reappraisal. Int. J. Cardiol. 2015, 180, $246-254$. [CrossRef] [PubMed]

23. Oh, S.; Goto, S.; Accetta, G.; Angchaisuksiri, P.; Camm, A.J.; Cools, F.; Haas, S.; Kayani, G.; Koretsune, Y.; Lim, T.W.; et al. Vitamin K antagonist control in patients with atrial fibrillation in Asia compared with other regions of the world: Real-world data from the GARFIELD-AF registry. Int. J. Cardiol. 2016, 223, 543-547. [CrossRef] [PubMed]

24. Phrommintikul, A.; Detnuntarat, P.; Prasertwitayakij, N.; Wongcharoen, W. Prevalence of atrial fibrillation in Thai elderly. J. Geriatr. Cardiol. 2016, 13, 270-273. [CrossRef] [PubMed]

25. Pengpid, S.; Peltzer, K. Underweight and overweight or obesity and associated factors among school-going adolescents in five ASEAN countries, 2015. Diabetes Metab. Syndr. 2019, 13, 3075-3080. [CrossRef] [PubMed]

26. Kongbunkiat, K.; Kasemsap, N.; Travanichakul, S.; Thepsuthammarat, K.; Tiamkao, S.; Sawanyawisuth, K. Hospital mortality from atrial fibrillation associated with ischemic stroke: A national data report. Int. J. Neurosci. 2015, 125, 924-928. [CrossRef]

27. Lee, S.R.; Choi, E.K.; Park, C.S.; Han, K.D.; Jung, J.H.; Oh, S.; Lip, G.Y.H. Direct Oral Anticoagulants in Patients with Nonvalvular Atrial Fibrillation and Low Body Weight. J. Am. Coll. Cardiol. 2019, 73, $919-931$. [CrossRef]

28. Malik, A.H.; Yandrapalli, S.; Shetty, S.; Aronow, W.S.; Jain, D.; Frishman, W.H.; Cooper, H.A.; Panza, J.A.; Investigators, M. Impact of weight on the efficacy and safety of direct-acting oral anticoagulants in patients with non-valvular atrial fibrillation: A meta-analysis. Europace 2020, 22, 361-367. [CrossRef]

29. Romero-Corral, A.; Montori, V.M.; Somers, V.K.; Korinek, J.; Thomas, R.J.; Allison, T.G.; Mookadam, F.; Lopez-Jimenez, F. Association of bodyweight with total mortality and with cardiovascular events in coronary artery disease: A systematic review of cohort studies. Lancet 2006, 368, 666-678. [CrossRef]

30. Lancefield, T.; Clark, D.J.; Andrianopoulos, N.; Brennan, A.L.; Reid, C.M.; Johns, J.; Freeman, M.; Charter, K.; Duffy, S.J.; Ajani, A.E.; et al. Is there an obesity paradox after percutaneous coronary intervention in the contemporary era? An analysis from a multicenter Australian registry. JACC Cardiovasc. Interv. 2010, 3, 660-668. [CrossRef]

31. Uretsky, S.; Messerli, F.H.; Bangalore, S.; Champion, A.; Cooper-Dehoff, R.M.; Zhou, Q.; Pepine, C.J. Obesity paradox in patients with hypertension and coronary artery disease. Am. J. Med. 2007, 120, 863-870. [CrossRef] [PubMed]

32. Momin, A.U.; Melikian, N.; Shah, A.M.; Grieve, D.J.; Wheatcroft, S.B.; John, L.; El Gamel, A.; Desai, J.B.; Nelson, T.; Driver, C.; et al. Leptin is an endothelial-independent vasodilator in humans with coronary artery disease: Evidence for tissue specificity of leptin resistance. Eur. Heart J. 2006, 27, 2294-2299. [CrossRef] [PubMed]

33. Higashi, Y.; Sasaki, S.; Nakagawa, K.; Kimura, M.; Noma, K.; Sasaki, S.; Hara, K.; Matsuura, H.; Goto, C.; Oshima, T.; et al. Low body mass index is a risk factor for impaired endothelium-dependent vasodilation in humans: Role of nitric oxide and oxidative stress. J. Am. Coll. Cardiol. 2003, 42, 256-263. [CrossRef]

34. Weber, M.A.; Neutel, J.M.; Smith, D.H. Contrasting clinical properties and exercise responses in obese and lean hypertensive patients. J. Am. Coll. Cardiol. 2001, 37, 169-174. [CrossRef]

35. Mak, K.H.; Bhatt, D.L.; Shao, M.; Haffner, S.M.; Hamm, C.W.; Hankey, G.J.; Johnston, S.C.; Montalescot, G.; Steg, P.G.; Steinhubl, S.R.; et al. The influence of body mass index on mortality and bleeding among patients with or at high-risk of atherothrombotic disease. Eur. Heart J. 2009, 30, 857-865. [CrossRef] 
36. Nakajima, K.; Yamaoka, H.; Morita, K.; Ebata, M.; Eguchi, S.; Muneyuki, T.; Munakata, H. Elderly people with low body weight may have subtle low-grade inflammation. Obesity (Silver Spring) 2009, 17, 803-808. [CrossRef]

37. Conway, D.S.; Buggins, P.; Hughes, E.; Lip, G.Y. Relationship of interleukin-6 and C-reactive protein to the prothrombotic state in chronic atrial fibrillation. J. Am. Coll. Cardiol. 2004, 43, 2075-2082. [CrossRef]

38. Shah, A.D.; Kandula, N.R.; Lin, F.; Allison, M.A.; Carr, J.; Herrington, D.; Liu, K.; Kanaya, A.M. Less favorable body composition and adipokines in South Asians compared with other US ethnic groups: Results from the MASALA and MESA studies. Int. J. Obes. (Lond.) 2016, 40, 639-645. [CrossRef]

(C) 2020 by the authors. Licensee MDPI, Basel, Switzerland. This article is an open access article distributed under the terms and conditions of the Creative Commons Attribution (CC BY) license (http://creativecommons.org/licenses/by/4.0/). 